Pino A., Chrofalo L. e Leonti F. Significatività e Randomizzazione e loro importanza in Zootecnia. Quaderni dell'Istituto di Zootecnia Generale dell'Università di Messina. Agosto 1957.

Raffaelit D. Variazione della tensione superficiale del latte. Annali di Chimica Applicata, vol. 30, p. 539, 1940.

RAHN. Da Mantovani G. (loco citato).

Rubinstein D. et Kusmina J. Da Pino A, e Chiofalo L. (loco citato).

Savini E. Caseificio : il latte e la sua produzione. 1946. Hoepli, Milano.

Schоlz. Da Montefredine A. (loco citato).

Sснмid. Da Montefredine A. (loco citato).

Schnorf. Da Montefredine A. (loco citato).

Segale M. Da Kopaczewski W. (loco citato).

Stassano. Da Mantovani G. (loco citato).

Tapernoux A. et Vuilladme E. Viscosité du lait de vache. Le Lait, tome XVI, p. $449,1934$.

Whitnah C. H. The Surface Tension of Milk. A Review. Journal of Dairy Science, vol. $42, \mathrm{n}^{\circ} 9$, p. $1437,1959$.

\title{
ÉTUDE-D'UNE MÉTHOdE DE RÉFÉRENCE POUR LA DÉTERMINATION DE LA MATIÈRE SÈCHE DANS LES FROMAGES : COMPARAISON AVEC LES MÉTHODES ACTUELLEMENT EN USAGE (1)
}

\author{
par \\ Mme MARTELLI
}

Ingénieur au Laboratoire Municipal de Paris

On entend par matière sèche d'un fromage, le résidu solide, entièrement déshydraté et constitué essentiellement par des matières protéiques et des matières grasses.

La matière sèche ainsi définie peut être déterminée soit par pesée du résidu après élimination totale de l'eau, soit par dosage de l'eau en tant que telle, les deux opérations étant complémentaires l'une de l'autre.

De la première technique relèvent des procédés couramment utilisés : séchage à l'étuve à $100^{\circ} \mathrm{C}$, séchage à $70-80^{\circ} \mathrm{C}$ sous une pression réduite, séchage sous vide à température ordinaire suivi d'une dessiccation à $100^{\circ} \mathrm{C}$ (méthode proposée par la Convention Internationale de Rome, 1954).

Le but recherché est atteint avec d'autant plus de difficulté que le fromage est plus riche en substances volatiles. Les méthodes

(1) Annales des Falsifications et de l'expertise chimique, 1962, $\mathrm{n}^{\mathrm{e}} 642,211$. 
de séchage à poids constant ou plus exactement à "perte faible constante " présentent un certain caractère d'incertitude et d'imprécision, car si la température choisie est trop élevée, il y a perte d'une partie de ces substances volatiles et si la température est trop basse, les protéines retiennent des quantités variables d'eau. D'autre part, un séchage trop prolongé provoque une augmentation de poids par oxydation de la matière grasse.

Théoriquement, le dosage de l'eau elle-même paraît donc plus satisfaisant et la méthode Karl-Fischer semble pouvoir apporter une solution à notre problème.

Mitchell et Smith, dans leur livre Aquametry (vol. V, 1948, Interscience Publishers Inc. I.T.P., London) signalent que RichteR a fait des déterminations de matière sèche dans le fromage "Edam " par la méthode Karl-Fischer (Angew-Chem., 48,776, 1935).

Malheureusement, la méthode Karl-Fischer ne permet de doser que de faibles quantités d'eau dans la prise d'essai, au maximum 80 à $100 \mathrm{mg}$, en utilisant une liqueur Fischer forte. Si donc, l'on veut doser directement par cette méthode, l'eau dont la teneur dans un fromage peut être de l'ordre de 50 p. 100, il faut limiter la prise d'essai à 150 ou $200 \mathrm{mg}$ au maximum. Une telle prise d'essai est trop faible pour être vraiment représentative d'un fromage.

Des essais ont été effectués au Laboratoire Municipal, par Mme Ruiz, qui a utilisé cette méthode en opérant sur une prise d'essai de 4 à $5 \mathrm{~g}$. La prise d'essai, introduite dans une fiole jaugée, était agitée en présence de méthanol, le dosage de l'eau était ensuite effectué sur une partie aliquote exactement mesurée. Mais, ainsi que Mme Rurz l'avait constaté, l'erreur commise sur le dosage de l'eau, dans la prise d'essai qui peut être de l'ordre de 1 à $2 \mathrm{mg}$, se trouve multipliée par un coefficient élevé (500), si l'on rapporte le résultat à $100 \mathrm{~g}$ de fromage.

Nous avons pensé qu'une prise d'essai de 2 à $3 \mathrm{~g}$ serait acceptable. Pour éviter d'opérer sur une partie aliquote, il devient donc indispensable de dessécher partiellement la prise d'essai, par un procédé simple excluant toute perte appréciable de substances volatiles, avant de déterminer l'eau restant par la méthode K. Fischer.

Nous avions constaté, en utilisant pour la détermination de la matière sèche, la méthode de la Convention Internationale de Rome, que, au cours de la dessiccation sous vide en présence d'acide sulfurique, le fromage perdait plus de 90 p. 100 de son humidité. Nous avons donc pensé à utiliser ce procédé de dessiccation préalable. 


\section{Description de la méthode}

\section{A. - Appareils et produits utilisés.}

Balance de précision.

Dessiccateur à vide de $25 \mathrm{~cm}$ de diamètre et $16 \mathrm{~cm}$ de hauteur utile.

Flacons cylindriques en verre de $10 \mathrm{~cm}$ de hauteur totale, $5 \mathrm{~cm}$ de diamètre extérieur, d'une capacité de $110 \mathrm{ml}$ environ et pesant $140 \mathrm{~g}$ environ. Les flacons sont munis d'un bouchon métallique pouvant se visser sur le col et permettant une fermeture hermétique.

Agitateurs en verre de $13 \mathrm{~cm}$ environ de longueur dont l'une des extrémités est aplatie et recourbée.

Petits agitateurs magnétiques.

Appareil de Karl Fischer.

Sable de Fontainebleau lavé aux acides et calciné.

Acide sulfurique concentré.

Méthanol pur anhydre.

Réactif de Fischer au 2-méthoxyéthanol (solution unique de la maison Touzart et Matignon) - $1 \mathrm{ml}$ de cette solution correspond à $7 \mathrm{mg}$ d'eau environ.

\section{B. - Mode opératoire.}

Introduire dans le flacon environ $4 \mathrm{~g}$ de sable et un agitateur.

Sécher le flacon à l'étuve et le peser après refroidissement dans un dessiccateur.

Introduire la prise d'essai comprise entre 2 et $3 \mathrm{~g}$ et pesée exactement.

Mélanger grossièrement la masse de fromage avec le sable à l'aide de l'agitateur.

Placer le flacon dans le dessiccateur à vide en présence d'acide sulfurique pendant au moins 16 heures (cette opération peut se faire avantageusement pendant la nuit).

Retirer le flacon du dessiccateur et, à l'aide de l'agitateur, écraser la matière partiellement desséchée afin de diviser finement la massè. (Etant donné la forme haute des flacons, cette opération s'effectue facilement sans perte.) Avec la pointe d'un couteau, détacher ce qui a pu adhérer à l'agitateur. A ce moment seulement, peser exactement la matière partiellement desséchée et immédiatement après introduire dans le flacon, $10 \mathrm{ml}$ de méthanol anhydre exactement mesurés en rinçant l'agitateur, et un petit agitateur magnétique. 
Boucher aussitôt le flacon à l'aide de son couvercle vissé.

Après 1 ou 2 heures de contact suivi de 30 minutes d'agitation, doser par la méthode Karl Fischer, l'eau ainsi extraite par le méthanol de cette matière partiellement desséchée.

On peut mener 8 à 10 déterminations simultanément et effectuer les titrations en série.

\section{C. - Calcul des résultats.}

Il suffit de déduire du poids de matière partiellement desséchée obtenue précédemment, la quantité d'eau restant qui vient d'être dosée (compte tenu de la petite quantité d'eau contenue dans $10 \mathrm{ml}$ de méthanol qui n'est jamais rigoureusement anhydre), pour obtenir le poids de matière sèche contenue dans la prise d'essai. Exemple :

$1^{\circ}$ Soit $2 \mathrm{~g}$, le poids de la prise d'essai de fromage ;

$2^{\circ}$ Soit $1 \mathrm{~g}$ le poids de matière partiellement desséchée que nous avons pesée après dessiccation sous vide;

$3^{\circ}$ Détermination de la correspondance entre le réactif de Fischer et la solution de méthanol hydraté.

(Nous utilisons toujours la méthode de Fischer avec titrage en retour de la liqueur de Fischer par le méthanol hydraté.)

Supposons que le réactif de Fischer et la solution de méthanol hydraté se correspondent exactement : soit $20 \mathrm{ml}$ réactif de Fischer $=20 \mathrm{ml}$ solution aqueuse de méthanol.

$4^{0}$ Détermination de la teneur en eau du méthanol anhydre (le méthanol pur n'est jamais rigoureusement anhydre).

Supposons que pour $10 \mathrm{ml}$ de méthanol anhydre, nous ayons ajouté $5 \mathrm{ml}$ de réactif de Fischer et $4,7 \mathrm{ml}$ de méthanol hydraté en retour : la quantité de réactif de Fischer correspondant à ces $10 \mathrm{ml}$ de méthanol anhydre est donc de 5,0-4,7=0,3 $\mathrm{ml}$.

50 Etalonnage du réactif de Fischer : soit $1 \mathrm{ml}$ de réactif de Fischer correspondant à $7 \mathrm{mg}$ d'eau.

$6^{\circ}$ Dosage de l'eau dans la matière partiellement desséchée que nous avons agitée avec $10 \mathrm{ml}$ de méthanol anhydre : soit $13,3 \mathrm{ml}$ de réactif de Fischer ajoutés et $3,0 \mathrm{ml}$ de méthanol hydraté versés en retour.

La quantité de réactif de Fischer qui a réagi est de : 13,3$3,0=10,3 \mathrm{ml}$ desquels il faut déduire $0,3 \mathrm{ml}$ correspondant aux $10 \mathrm{ml}$ de méthanol anhydre ajoutés. 
Nous avons donc finalement $10,3-0,3=10,0 \mathrm{ml}$ de réactif de Fischer ayant réagi pour la prise d'essai.

La quantité d'eau correspondante est de $7 \times 10=70 \mathrm{mg}$.

La matière sèche contenue dans la prise d'essai de $2 \mathrm{~g}$ de fromage est de $1000-70=930 \mathrm{mg}=0,930 \mathrm{~g}$ d'où :

Matière sèche dans $100 \mathrm{~g}$ de fromage $: \frac{0,930 \times 100}{2}=46,5 \mathrm{~g}$.

Précision de la méthode. - Nous avons constaté au cours de ces essais qu'en effectuant de 4 à 6 déterminations sur un même fromage, les écarts maximals entre les résultats rapportés à $100 \mathrm{~g}$ de fromage étaient de l'ordre de 0,4 dans les plus mauvais cas.

Critique de la méthode. - Apparemment, le dosage de la matière sèche ainsi effectué, ne semble pas critiquable : l'évaporation de la majeure partie de l'eau à la température ordinaire ne risque pas de faire perdre des substances volatiles et la méthode de K. Fisscher ne dose que l'eau restante.

Toutefois, dans le cas d'un fromage à réaction fortement ammoniacale ou acide, on pourrait craindre que la dessiccation sous vide ne provoque des pertes de produits volatils (ammoniac ou acide gras volatils). Nous avons donc effectué quelques essais dans lesquels nous avons neutralisé la prise d'essai, en présence d'un indicateur, par la soude ou l'acide sulfurique titrés suivant que le fromage était acide ou alcalin. Pour cela, la prise d'essai a été au préalable malaxée avec $10 \mathrm{ml}$ d'acétone. Dans l'un et l'autre cas, nous avons obtenu des résultats très voisins avec ou sans neutralisation. Cette neutralisation est d'ailleurs très difficile à réaliser avec exactitude, le virage étant très délicat à saisir. De plus, la dessiccation sous vide est alors plus laborieuse et il faut parfois la prolonger pendant 48 heures ou même davantage, pour que la quantité d'eau restante puisse être ensuite dosée par la méthode K. Fischer.

Nous avons comparé les résultats obtenus pour la détermination de la matière sèche dans les fromages par la méthode Karl Fischer, avec ceux obtenus par la méthode de la Convention Internationale de Rome, à laquelle nous avons apporté de légères modifications.

Rappelons cette méthode. - Dans une capsule ou un cristallisoir de $70 \mathrm{~mm}$ de diamètre, on introduit environ $20 \mathrm{~g}$ de sable et un agitateur de verre dont l'une des extrémités est aplatie et recourbée. On sèche le matériel à l'étuve et on introduit environ $5 \mathrm{~g}$ de fromage exactement pesés que l'on mélange soigneusement avec le sable. L'échantillon ainsi préparé est d'abord desséché, à la température 
TABLEAU I

FROMAGE A L'ÉTAT FRAIS

Matière sèche en gramme pour 100 grammes

\begin{tabular}{|c|c|c|c|}
\hline Désignation du fromage & $\begin{array}{l}\text { Méthode } \\
\text { Fischer }\end{array}$ & $\begin{array}{c}\text { Méthode } \\
\text { Convention } \\
\text { Internatoinale } \\
\text { de Rome }\end{array}$ & $\begin{array}{l}\text { Méthode Etuve } \\
\qquad 0^{\circ} \\
\text { sous pression } \\
\text { réduite }\end{array}$ \\
\hline Camembert I ........... & 45,6 & 45,0 & 45,2 \\
\hline Camembert II $\ldots \ldots \ldots \ldots$ & 49,2 & 48,8 & 49,4 \\
\hline Camembert III $\ldots \ldots \ldots \ldots \ldots$ & 58,5 & 58,5 & 58,8 \\
\hline Brie $\ldots \ldots \ldots \ldots \ldots \ldots \ldots$ & 50,0 & 49,9 & 50,0 \\
\hline Saint-Marcellin .......... & 49,6 & 50,0 & 50,0 \\
\hline Chèvre I . . . . . . . . . . . . & 53,4 & 53,7 & 54,2 \\
\hline Chèvre II $\ldots \ldots \ldots \ldots \ldots \ldots$ & 58,8 & 58,4 & 58,8 \\
\hline Port-Salut $I \quad \ldots \ldots \ldots \ldots \ldots$ & 56,0 & 56,5 & 57,3 \\
\hline Port-Salut II . . . . . . . . & 54,7 & 55,0 & 55,4 \\
\hline Gruyère I $\ldots \ldots \ldots \ldots \ldots$ & 63,7 & 63,5 & 64,5 \\
\hline Gruyère II $\ldots \ldots \ldots \ldots \ldots \ldots$ & 65,3 & 65,3 & 65,7 \\
\hline Hollande I . . . . . . . . . . & 56,4 & 56,5 & 57,3 \\
\hline Hollande II $\ldots \ldots \ldots \ldots \ldots$ & 61,2 & 61,4 & 62,0 \\
\hline Bonbel $\ldots \ldots \ldots \ldots \ldots \ldots$ & 55,7 & 56,0 & 56,6 \\
\hline Munster I $\ldots \ldots \ldots \ldots \ldots$ & 46,9 & 46,6 & 46,7 \\
\hline Munster II ............ & 51,0 & 51,0 & 51,3 \\
\hline Edam I $\ldots \ldots \ldots \ldots \ldots \ldots$ & 56,9 & 57,0 & 57,7 \\
\hline Edam II $\ldots \ldots \ldots \ldots \ldots \ldots$ & 58,0 & 58,0 & 58,5 \\
\hline Pont-l'Evêque ........... & 55,0 & 54,9 & 55,3 \\
\hline 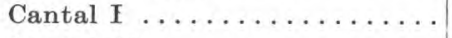 & 61,4 & 61,6 & 61,8 \\
\hline Cantal II $\ldots \ldots \ldots \ldots \ldots \ldots$ & 59,8 & 59,9 & 60,4 \\
\hline Reblochon $\ldots \ldots \ldots \ldots \ldots$ & 49,4 & 49,3 & 49,4 \\
\hline Bleu d'Auvergne I . . . . . . . & 62,0 & 61,0 & 61,4 \\
\hline Bleu d'Auvergne II $\ldots \ldots \ldots$ & 60,5 & 60,1 & 60,4 \\
\hline Bleu d'Auvergne III $\ldots \ldots \ldots$ & 61,8 & 62,0 & 62,3 \\
\hline Roquefort I ............ & 63,3 & 62,7 & 63,1 \\
\hline Roquefort II $\ldots \ldots \ldots \ldots \ldots$ & 61,3 & 60,9 & 61,5 \\
\hline Crème de Gruyère $\ldots \ldots \ldots \ldots$ & 46,2 & 45,9 & 46,4 \\
\hline Demi-sel ............... & 33,6 & 33,5 & 33,9 \\
\hline Demi-Suisse $\ldots \ldots \ldots \ldots \ldots$ & 26,6 & 26,7 & 27,0 \\
\hline
\end{tabular}

ordinaire, dans un dessiccateur à vide, en présence d'acide sulfurique, pendant au moins 16 heures. Il est ensuite desséché à l'étuve à $100^{\circ} \mathrm{C}$. On poursuit la dessiccation pendant au moins 6 heures et on procède ensuite aux pesées à courts intervalles ( $1 / 2$ heure) jusqu'à poids constant. Nous avons effectué la première 
matière sèche déterminée par notre méthode, environ de 1 p. 100. En effet, les acides gras volatils particulièrement abondants dans ce fromage sont susceptibles d'être en partie éliminés par le chauffage à $100^{\circ}$ à l'étuve prévu pour la méthode de la Convention Internationale de Rome.

2 o D'autre part, il semble que dans bien des cas, la méthode de séchage à l'étuve à $70^{\circ}$, sous pression réduite, donne des résultats trop élevés, surtout avec les fromages cuits ou à pâte pressée. Ce que nous constatons avec le Gruyère I, le Hollande, le Bonbel, le Port-Salut, l'Edam I. De plus, très souvent, la dessiccation traîne en longueur. Il n'est en effet, pas surprenant que dans ces fromages à pâte assez dure, l'eau soit particulièrement retenue par les protéines. Toutefois, dans le cas des fromages plus ou moins avariés, cette méthode donne des résultats supérieurs à ceux de la méthode de la Convention Internationale de Rome et très voisins de ceux obtenus par la méthode K. Fischer.

\section{Summary}

On examining results we have obtained with these tests, it appears that the following conclusions can be drawn :

1) In the case of cheese, in a fresh condition or well preserved, the method proposed by the International Convention of Rome as well as the method developed by K. Fischer and adapted to the determination of solids in cheese give very similar results. In a few cases, we have obtained with mouldy soft cheeses significant deviations, especially with a Bleu d'Auvergne, in which the solids determined by the method of the International Convention of Rome were lower than the solids determined by our method, namely about 1 per cent. In fact, volatile fatty acids which are quite plentiful in this sort of cheese, are likely, to be partially eliminated under the influence of heating in the oven at $100^{\circ} \mathrm{C}$ $\left(212^{\circ} \mathrm{F}\right)$, procedure provided for in the method of the International Convention of Rome.

2) On the other hand, it appears that the method of drying in the oven at $70^{\circ} \mathrm{C}\left(158^{\circ} \mathrm{F}\right)$ under reduced pressure give most often too high results, chiefly with cooked or pressed cheeses. It is exactly what we have observed with Gruyère I, Hollande, Bonbel, Port-Salut and Edam I. Moreover, it occurs very frequently that dessication takes a long time. The fact that water can be readily retained by proteins is not surprising with these 
rather hard cheeses. However, in the case of more or less damaged cheeses, this method give results which are higher than those obtained by the method of the International Convention of Rome, and very similar to the data obtained with the method developed by K. FIscher.

\title{
RECHERCHES SUR LA PRÉSENCE DE « MYCOBACTÉRIES », TYPES HOMINIS ET BOVIS, DANS LE LAIT DE MARCHÉ A VARSOVIE
}

par

\author{
W. MACANDER-GOLCZ
}

Chef du Laboratoire de la tuberculose Station sanitaire et épidémiologique. Varsovie

Il est bien connu que Mycobacterium, type bovis, est très souvent l'agent de la tuberculose chez l'homme et surtout chez les enfants nourris de lait cru, ce qui est malheureusement le cas, encore très fréquent, principalement dans les faubourgs de Varsovie et d'autres grandes villes de Pologne.

Le lait des femelles de l'espèce bovine qui sont tuberculeuses, contient des bacilles tuberculeux, qu'il existe ou non des lésions de la mamelle, même si l'infection tuberculeuse ne se traduit pas par d'autres signes que la réaction à la tuberculine [1], [3], [4], [5], [6], [7], [8], [9]. D'autre part, le lait peut contenir des bacilles tuberculeux humains apportés par ceux qui le manipulent et, en particulier, par le trayeur.

En outre, nous avons voulu étudier l'influence saisonnière sur le nombre des bacilles tuberculeux du lait, étant donné que la température atmosphérique et la nourriture des vaches ont une répercussion sur le nombre de ces germes.

Pour avoir des résultats sûrs, nous avons utilisé à la fois des méthodes bactériologiques et biologiques [12] [13] [14] [15] [16] [17].

Nous avons ainsi étudié 348 échantillons de lait de marché, prélevés en bouteilles stériles de $500 \mathrm{ml}$ et $200 \mathrm{ml}$. Le lait fut centrifugé 30 minutes à 3000 tours. Les frottis de la crème mélangée avec le sédiment furent colorés par la méthode de Ziehl-Nielsen et examinés au microscope.

Le mélange fut également homogénéisé avec $\mathrm{NaOH}$ à 4 p. 100, pendant 30 minutes à $37^{\circ} \mathrm{C}$, puis neutralisé par $\mathrm{HCl}$ à $4 \mathrm{p}$. 100 , ensemencé sur les milieux de Loevenstein-Jensen et de Petragnani ; en même temps, nous avons préparé une microculture par 